- Canadá solo ha presentado una reclamación parcial de plataforma continental extendida relativa al Océano Atlántico el 6 de diciembre de 2013. Dados sus ambiciosos objetivos, todo hace sospechar que su solicitud de plataforma continental extendida en el Ártico será muy extensa y lo más probable es que se solape con la de la Federación de Rusia, Dinamarca y Estados Unidos.

- En un Acuerdo Provisional de 2006 (Agreed Minutes) concluido entre Islandia, Noruega y Dinamarca (Islas Feroe), los tres Estados establecen un principio de acuerdo sobre cómo proceder en el Mar de Noruega con sus peticiones solapadas de plataforma continental extendida, estableciendo un acuerdo tripartito que, para culminarse, precisa que la labor recomendatoria de la CLPC se termine.

- Noruega presentó su solicitud de plataforma continental extendida ante la CLPC en 2006, relativa al Loophole en el Mar de Barents, la Cuenca Nansen Oeste en el Océano Ártico y el Banana Hole en el Mar de Noruega, y desde abril de 2009 ha sido el primer país en obtener recomendaciones de la CLPC.

- Estados Unidos no es parte de la CNUDM y, por tanto, no puede entrar en el proceso de la CLPC. No obstante, la política norteamericana respeta los fundamentos del art. 76 de la CNUDM. Caben varias posibilidades: a) si los Estados Unidos delimitan su plataforma continental extendida sin el beneplácito de la CLPC, pueden encontrarse con la oposición de terceros que no respeten tal acto unilateral como válido; $b$ ) ante tal contingencia, los Estados Unidos se plantean pasar a ser parte de la CNUDM; c) cabría la posibilidad de llegar a un acuerdo negociado de límites con sus contrapartes (tal y como prevé en último término los arts. 76.8 y 76.10 de la CNUDM).

7. Las escasas controversias territoriales en el espacio ártico, los múltiples acuerdos, soluciones consensuadas o respeto a decisiones judiciales en lo que se refiere a las controversias marítimas, revelan que el Ártico ha funcionado como un espacio de cooperación pacífica. Por el momento, la actividad política que rodea a las reclamaciones de plataforma continental extendida en el Ártico, hace pensar más en invitaciones a la negociación conjunta de límites en zonas de solapamiento de dichas plataformas, que a agresivas proclamas soberanistas.

Elena CONDE PÉREZ

Universidad Complutense de Madrid http://dx.doi.org/10.17103/redi.68.1.2016.4a.04

\title{
5. LA SENTENCIA DEL TRIBUNAL EUROPEO DE DERECHOS HUMANOS EN EL CASO VASILIAUSKAS C. LITUANIA (GS): EL GRUPO PROTEGIDO EN EL CRIMEN DE GENOCIDIO Y SU LESIÓN EN EL MARCO DEL ART. 7 DEL CONVENIO EUROPEO PARA LA PROTECCIÓN DE LOS DERECHOS Y DE LAS LIBERTADES FUNDAMENTALES
}

1. Previo a cualquier análisis sobre la Sentencia Tribunal Europeo de Derechos Humanos (TEDH) en el caso Vasiliauskas c. Lituania [GS] (núm. 35343/05, Sentencia de 20 de octubre de 2015), es importarte recordar que la trágica historia de las Repúblicas Bálticas a partir de la Segunda Guerra Mundial permite comprender, en gran parte, el modo en que recogieron en sus Códigos Penales el delito de genocidio tras su independencia de la Unión Soviética. Aunque la concreta regulación en estos tres Estados no ha sido idéntica, a grandes rasgos puede afirmarse que han compartido un carácter expansivo que favorecía la calificación de genocidio de los crímenes cometidos en aquella época, y muy especialmente de los perpetrados durante la ocupación soviética. En el asunto que ahora interesa, de hecho y por si pudiera quedar 
alguna duda, la primera norma lituana al respecto, de 9 de abril de 1992, indicaba expresamente en su art. 2 que los asesinatos, torturas y deportaciones de habitantes de Lituania cometidos en la ocupación nazi y soviética se considerarían un crimen de genocidio tal y como lo define el Derecho internacional. En otros casos, como el Código Penal estonio de 2001, se optó por incluir junto a los grupos protegidos que figuran en la Convención para la Prevención y la Sanción del Delito de Genocidio, a quienes «resisten la ocupación»; previsión que no existía como tal en el posterior Código Penal lituano de 2003, que no obstante y de forma similar a la normativa letona, sí incluía en su art. 99 a los grupos «sociales o políticos» entre ellos.

2. Esta breve introducción nos dirige, en primera instancia, hacia uno de los temas más intensamente debatidos desde la misma redacción de la Convención contra el Genocidio y hasta la fecha, cual es, el conocido como "genocidio político». Es decir, si el exterminio de grupos políticos constituye, o no, un crimen de genocidio. De hecho, el demandante en el caso que examinamos - miembro del Ministerio de Seguridad del Estado y después de la no menos funesta KGB - había sido condenado por el tribunal regional de Kaunas, en febrero de 2004, por haber participado en 1953 en el exterminio de los habitantes de Lituania que pertenecían a «un grupo político separado» que se resistía a la ocupación soviética; crimen que fue calificado como genocidio. Ahora bien, poco después el tribunal de apelaciones precisó sobre lo anterior que los miembros de ese grupo tenían al mismo tiempo la condición de representantes de la nación lituana, es decir, de un «grupo nacional»; concluyendo, en suma, que los partisanos lituanos debían entenderse como un grupo no solo político, sino también a nacional y/o étnico. Es decir, dos de los grupos protegidos que expresamente figuran en la Convención contra el Genocidio. Por su parte y en lo que aquí nos ocupa, en febrero de 2005 el Tribunal Supremo sostendría que los partisanos encarnaron la resistencia de la «nación lituana» contra la ocupación soviética. Años después, el Tribunal Constitucional (re)formularía lo resumido indicando que, de acuerdo con las normas internacionales universalmente reconocidas, las acciones llevadas a cabo contra ciertos grupos políticos y sociales lituanos podían ser consideradas genocidio si tenían como objeto la destrucción de quienes representaban una parte importante de la nación lituana, amenazando así a la misma supervivencia de toda ella [Vasiliauskas c. Lituania (GS), párrs. 30-40 y 56-63].

De este modo, de la mano de los conceptos de «grupo político» y de extermino «parcial o en parte» de un "grupo nacional», fueron dos las cuestiones que debió enfrentar la Gran Sala del TEDH en el caso Vasiliauskas. No atendiendo a su configuración o contenido actuales, sino a la consideración del genocidio en 1953; esto es, como ya dijimos, el año en que se cometieron los hechos concretos. Y es que a juicio del demandante, en cualquiera de los dos casos lo resumido había supuesto una violación del art. 7 del Convenio Europeo para la Protección de los Derechos y de las Libertades Fundamentales (CEDH); en tanto que en esencia, se le había aplicado una tipificación del genocidio que excedía de lo que el Derecho internacional considerada como tal en aquel entonces. A este respecto, valga adelantar entonces que además de lo dicho, sería un elemento clave la general exigencia mantenida por el TEDH bajo lo que ha venido denominando "condiciones cualitativas», como las de accesibilidad y previsibilidad. Expresado en términos básicos, se trataría de que la aplicación del delito en cuestión ha de ser previsible para el sujeto [véase, por ejemplo, Korbely c. Hungría (GS), núm. 9174/02, Sentencia de 19 de septiembre de 2008, párr. 70].

3. Como es sabido, no ha sido esta la primera vez en que el TEDH ha abordado la delimitación del crimen de genocidio (véase Jorgic c. Alemania, núm. 74613/01, 
Sentencia de 12 de julio de 2007); tampoco la aplicación del art. 7.2 del CEDH a escenarios posteriores a la Segunda Guerra Mundial (consúltense, Kolk y Kislyiy c. Estonia, núms. 23052/04 y 24018/04, Decisión de 17 de enero de 2006, y Penart c. Estonia, núm. 14685/04, Decisión de 24 de enero de 2006). Sobre este segundo particular, tras estas dos decisiones, ciertamente la Gran Sala asumió una postura más restrictiva [véase especialmente, Kononov c. Letonia (GS), núm. 36376/04, Sentencia de 17 de mayo de 2010]; pero, en cualquier caso, en la Sentencia Vasiliauskas este asunto tuvo un protagonismo insignificante, siendo ventilado en apenas unos párrafos (en general, párrs. 187-190, que en realidad solo refieren a la posición anterior de la Gran Sala). Por ello, en esta oportunidad no entraremos en esta interesante cuestión general.

4. Analizando entonces el examen que realizó el TEDH sobre el crimen de genocidio, cabe también hacer una distinción a efectos expositivos, siguiendo lo señalado supra. En primer lugar, en lo que respecta al "genocidio político», la posición de la Gran Sala se apoyó singularmente en decisiones cercanas como la del Tribunal Internacional de Justicia, esto es, en la afirmación de que ya en el mismo proceso de redacción de la Convención contra el Genocidio, fue capital la identificación positiva de los grupos con características específicas para decidir qué grupos se iban a incluir y cuáles, como los grupos políticos, quedarían excluidos [Caso Relativo a la Aplicación de la Convención para la Prevención y la Sanción del Delito de Genocidio (Bosnia-Herzegovina c. Serbia y Montenegro), ICJ Reports 2007, párr. 194]. Agregando que todas las normas convencionales posteriores - hasta el Estatuto de la Corte Penal Internacional - no se habían separado de lo anterior; con lo que aunque algunos Estados hubieran decidido criminalizar el genocidio de un grupo político en sus leyes nacionales, no cabía entender en ningún caso que en 1953 ello fuese así de conformidad al Derecho internacional — convencional o general- [Vasiliauskas c. Lituania (GS), párrs. 170-175].

En este punto y en el asunto de fondo, entre las diversas posiciones críticas siempre cabría recordar, a modo de síntesis, interrogantes como aquel que entre nosotros se formulase en 1951; esto es: "¿Por qué [...] los grupos políticos de un país pueden ser exterminados sin que los autores de tales hechos aparezcan incriminados como genocidas?» (MiaJA DE LA MuELA, A., «El genocidio, delito internacional», REDI, 1951, núm. 2, pp. 376-377). No obstante, la conclusión ofrecida por el TEDH fue la generalmente admitida, y de hecho y a tenor de los votos particulares a la sentencia, no planteó discrepancia mayor.

5. Muy diferente fue lo que podemos considerar como segunda cuestión, no solo singularmente más compleja sino especialmente polémica en lo particular del caso, pues puede decirse que partió al tribunal casi en dos bloques. En lo jurídico, su esencia cabe dirigirla al debate acerca de los criterios que permitan fijar los límites de la parte del grupo cuya destrucción se pretende (FERnández PACHECo, C., El genocidio en el Derecho Penal Internacional, Valencia, Tirant lo Blanch, 2011, pp. 179-193). Particular que enlaza con el mismo sentido de la formulación «destruir en todo o en parte» (Schabas, W. A., Genocide in International Law: The Crime of Crimes, Cambridge, 2. ${ }^{a}$ ed., Cambridge University Press, 2009, pp. 273 y ss.); y que conduce hasta la propia concepción general del bien jurídico protegido y de su lesión; es decir, hasta el debate acerca de si la destrucción del grupo debe ser entendida en una dimensión física o biológica (GIL GIL, A., Derecho penal internacional. Especial consideración del delito de genocidio, Madrid, Tecnos, 1999, pp. 160 y ss.) o también social como mantiene, por ejemplo, parte de la doctrina alemana remitiéndose a la idea original de Lemkin (Werle, G. y Jessberger, F., Principles of International Criminal Law, Oxford, 3. a ed., 
Oxford University Press, 2014, p. 318). En lo más concreto y de su lado, nos enfrentaríamos también a lo que se ha calificado como la "mayor controversia» en este ámbito: la interpretación del elemento normativo "grupo nacional» (OllÉ SeSÉ, M., Justicia universal para crímenes internacionales, Madrid, La Ley, 2008, p. 491).

6. Con todo, la tesis sostenida por nueve de los 17 jueces de la Gran Sala vino a mantener que, en atención a los trabajos preparatorios y a la finalidad de la Convención contra el Genocidio, la mención de su art. II (la intención de destruir en parte a un grupo nacional, en este caso) solo cabía entenderla como referida a criterios cuantitativos, esto es, a la voluntad de exterminar una parte sustancial de grupo —en términos numéricos-. En este punto, obviamente el TEDH era consciente del conjunto de decisiones judiciales — cuya gran parte cita— que han puesto el acento en los criterios cualitativos, esto es, en lo que se suele conocer como la intención de destruir a una «parte prominente o significativa» del grupo. $\mathrm{Al}$ respecto, conviene apuntar que, al menos en mi opinión, parte de esta jurisprudencia es un tanto confusa en lo que se refiere a la distinción y/o relación entre la parte del grupo —o subgrupo significativo o prominente- y el grupo en su totalidad, pero dentro del espacio ahora disponible, baste ilustrar esta segunda postura con un par de referencias.

7. La primera, muy similar a la que suele considerarse como su inicial plasmación escrita (Doc. ONU S/1994/674), la del Tribunal ad hoc para la ex Yugoslavia. A saber: si no se cumple el criterio cuantitativo, la intención de destruir «en parte» puede establecerse si hay pruebas de que la destrucción se ha dirigido a una parte significativa del grupo, tal como sus líderes; siendo importante en este sentido, que la eliminación de estas personas tenga un impacto sobre la supervivencia del grupo como tal (Fiscalía c. Sikirica, IT-95-8, Sentencia de 3 de septiembre de 2001, párrs. 7677). La segunda, más reciente y seguramente algo más matizada, la del Tribunal Internacional de Justicia, que recordó en lo que nos ocupa que: «Account must also be taken of the prominence of the allegedly targeted part within the group as a whole» [Caso Relativo a la Aplicación de la Convención para la Prevención y la Sanción del Delito de Genocidio (Croacia c. Serbia), ICJ Reports 2015, párr. 142].

Ahora bien, para la mayoría del TEDH lo determinante fue que esta interpretación, sobre cuyo acierto no se manifestó expresamente, era muy posterior a 1953. De tal suerte que a su juicio no resultaba posible entender que el exterminio de aquellos lituanos que se oponían a la ocupación soviética fuera encuadrable como una serie de hechos dirigidos a destruir (parcialmente) al grupo nacional lituano. Resultando que el demandante no podría haber previsto entonces que el asesinato de los partisanos lituanos podría constituir un genocidio de los nacionales de Lituania; ni siquiera con la ayuda de un abogado, aclaró. A todo ello se añadió que además y en cualquier caso, incluso si la posterior interpretación cualitativa de los tribunales internacionales estuviera ya establecida y fuera previsible en 1953, los tribunales lituanos no habían demostrado suficientemente en lo fáctico, porqué o sobre qué base en aquel año los partisanos lituanos constituían una parte importante del «grupo nacional» [Vasiliauskas c. Lituania (GS), párrs. 165-186].

Esta última valoración fue singular y duramente criticada en votos disidentes como el del Juez Kuris. Pero en la cuestión jurídica general, la minoría reconocería que en 1953 no existía un desarrollo jurisprudencial acerca del significado de la frase "destruir en parte», si bien, esgrimió algo cuyo sustento fundamental conviene citar: «The fact that judicial interpretation of the 1948 Genocide Convention took time to develop cannot in itself mean that genocide did not occur prior to such interpretation" (voto disidente de los Jueces Villiger, Power-Forde, Pinto de Albuquerque y Kuris, 
párr. 18). La idea capital, que como tal aparece en el voto disidente de la Juez Ziemele (párr. 17), podría resumirse en que las decisiones judiciales que vinieron a explicitar lo antes dicho, no se corresponderían con nuevas interpretaciones de la Convención contra al Genocidio, en el sentido de incorporar nuevos elementos que no estaban presentes en ella, sino que se dieron en aquellos años porque, en fin, fue entonces cuando se abordó este crimen en sede judicial. Y a partir de ello, consideraron también infundada la conclusión de que resultase imposible para el demandante prever que estaba cometiendo un crimen de genocidio. Es decir, en lo más concreto, la posición fue que en tanto que en aquel mismo 1953 el demandante había declarado que como agente del Ministerio de Seguridad del Estado compartía el objeto de exterminar a los miembros de la resistencia nacionalista lituana [Vasiliauskas c. Lituania (GS), párr. 18], era probable que «un asesor legal independiente» le hubiera advertido de que ello tenía las características esenciales del crimen de genocidio (voto disidente de los Jueces Villiger, Power-Forde, Pinto de Albuquerque y Kuris, párr. 30).

8. Algunos autores han sostenido que la posición defendida por la mayoría del TEDH responde a una suerte de "evaluación estricta» sobre el requisito de la previsibilidad, avanzando que de ello pueden derivarse diversos problemas en otros casos o instancias (SKander GALAND, A., "The Strasbourg Court Destroys "in part" The Hague's Case Law on Genocide», en https://kuremer.ku.edu.tr/en/strasbourg-court-destroys-part-hagues-case-law-genocide). Ciertamente, no cabe referir la crítica hacia la defensa de interpretaciones excesivamente expansivas cuando se trata de las importantes garantías que implica el art. 7 del $\mathrm{CEDH}$, pero la postura del tribunal plantea no pocas cuestiones espinosas. Sin duda, desde una dimensión histórica y/o política (véase, por ejemplo, Human Rights Monitoring Institute, «Is Killing Freedom Fighters Genocide? Lithuania Fails to Prove Claim», en http://www.liberties.eu/en/news/ lithuania-genocide-political-group), aspecto que no se debe minusvalorar si se presta atención al modo en que esta sentencia ha sido interpretada por algunos medios de comunicación rusos (véase, por ejemplo, SHAmshiev, A., «The European Court is Against the Lithuanian National Myth», en http://www.rubaltic.ru/articles/lithuaniannational-myth-27102015/); pero también y especialmente en lo jurídico. Limitémonos ahora a destacar una de ellas: Si en 1953 aún no estaban establecidos los criterios cualitativos en lo que al genocidio respecta, ¿cuándo quedaron así determinados? Pues si la respuesta fuera que no hasta finales de la década de los noventa del siglo pasado, parece evidente que a todas las personas a las que se les hubiesen aplicado por crímenes cometidos con anterioridad, se les habría violado el mismo derecho que al demandante en el caso Vasiliauskas...

Javier CHINCHÓN ÁLVAREZ Universidad Complutense de Madrid http://dx.doi.org/10.17103/redi.68.1.2016.4a.05

\section{LA CRISIS MIGRATORIA Y LA REINSTAURACIÓN DE LOS CONTROLES DE LAS FRONTERAS INTERIORES EN EL ESPACIO SCHENGEN}

1. La denominada crisis de los refugiados ha implicado el traslado de centenares de miles de personas desde zonas en conflicto, especialmente, aunque no exclusivamente, procedentes de Siria, Iraq, Afganistán y Libia hacia la Unión Europea (UE) en busca de protección internacional. Este desplazamiento se realiza sorteando innumerables obstáculos, en condiciones lamentables y con grave riesgo para las vidas de los participantes. La magnitud de esta marea humana está afectando seriamente a la 